\title{
Estudo da ocorrência de enteroparasitas em hortaliças comercializadas na região metropolitana de São Paulo - SP, Brasil. II - Pesquisa de protozoários intestinais*
}

\author{
Study of the occurrence of intestinal parasites os vegetables comercially \\ traded in the metropolitan area of S.Paulo, SP - Brazil. II - Research into \\ protozoan cysts
}

\author{
Carlos Augusto Fernandes de Oliveira*, Pedro Manuel Leal Germano
}

\begin{abstract}
OLIVEIRA, C.A.F. DE \& GERMANO, P.M.L. Estudo da ocorrência de enteroparasitas em hortaliças comercializadas na região metropolitana de São Paulo - SP, Brasil. II - Pesquisa de protozoários intestinais. Rev.Saúde públ., S.Paulo, 26: 332-5, 1992. Foram analisadas hortaliças "in natura", comercializadas na Região Metropolitana de São Paulo, SP-Brasil, visando à pesquisa e à identificação de cistos de protozoários de interesse médico. As hortaliças, constituídas de 50 amostras de cada variedade, consistiram em: alface (Lactuca sativa), variedades lisa e crespa, escarola (Chichorium sp) e agrião (Nasturtium officinale). Os resultados evidenciaram elevados percentuais de contaminação em todas as variedades de hortaliças analisadas, porém as freque ências de protozoários foram maiores no agrião. As amostras de alfaces apresentaram os menores percentuais de contaminaçâo, enquanto que a escarola apresentou valores geralmente situados entre o agrião e as alfaces. Observou-se uma grande variedade de protozoários, cujas frequeências de ocorrência na população residente na Região Metropolitana de São Paulo são igualmente elevadas. Os mais freqũentes foram Entamoeba sp (com 4 e 8 núcleos) e Giardia sp. Foram também isolados cistos de Iodamoeba sp, Endolimax $s p$ e Chilomastix sp. Os elevados níveis de contaminação fecal encontrados nas amostras analisadas apontam para a importância dos alimentos na transmissão de protozoários intestinais.
\end{abstract}

Descritores: Vegetais, análise. Protozoários, isolamento. Contaminação de alimentos, análise.

\section{Introdução}

As enfermidades intestinais causadas por protozoários constituem sério problema de saúde pública em diversos países, inclusive o Brasil, onde os coeficientes de prevalência de algumas protozooses ainda são consideravelmente elevados ${ }^{8,11}$.

Neste contexto, sobressai a importância que as hortaliças, principalmente aquelas consumidas in natura, podem desempenhar como vias de transmissão de protozoários intestinais ${ }^{6}$, uma vez que, no Brasil, esses alimentos freqüentemente são adubados com dejetos humanos, ou irrigados com águas contaminadas com matéria fecal ${ }^{7}$.

* Extraido da Dissertação de Mestrado "Estudo da ocorrência de enteroparasitas em hortaliças comercializadas na região metropolitana de São Paulo, SP - Brasil", apresentada à Faculdade de Saúde Pública da USP, em 1991.

** Secretaria Municipal de Abastecimento de São Paulo, SP - Brasil.

*** Departamento de Prática de Saúde Pública da Faculdade de Saúde Pública da USP - São Paulo, SP - Brasil

Separatas/Reprints: P.M.L. Germano - Av. Dr. Amaldo, 715 - 01246-904 - São Paulo, SP - Brasil.

Publicação financiada pela FAPESP. Processo Saúde Coletiva 91/4994-0
Apesar da relevância do tema em questão, sāo poucos os trabalhos nacionais teferidos na literatura médica que estabelecem graus de contaminação de hortaliças por protozoários intestinais. Hernandez e col. ${ }^{4}$ (1981) encontraram cistos de Entamoeba sp (com 4 e 8 núcleos), Giardia $s p$ e Iodamoeba $s p$ em amostras de hortaliças provenientes de 12 hortas localizadas no Município de Biritiba Mirim - SP, um dos principais produtores de verduras do Estado. Mota e col. ${ }^{6}$ (1982), em Curitiba - PR, e Miche e Morganti ${ }^{5}$ (1983), em São Paulo - SP, atestaram igualmente a ocorrência de protozoários em hortaliças, em altos percentuais.

Com base nestes aspectos, costituem objetivos do presente trabalho:

- pesquisar, qualitativametne, a presença de cistos de protozoários de interesse médico em amostras de quatro tipos de hortaliças, provenientes de diferentes produtores do Estado de São Paulo e comercializadas na Companhia de Entrepostos e Armazéns Gerais de São Paulo (CEAGESP);

- estabelecer as possíveis diferenças entre os percentuais de contaminação dos tipos de hortaliças selecionadas. 


\section{Material e Método}

Utilizaram-se os critérios adotados por Oliveira e Germano ${ }^{7}$ (1992), estabelecendo-se em 50 o número de amostras para cada tipo de hortaliça: alface (Lactuca sativa), variedade lisa e crespa, escarola (Chicorium sp) e agrião (Nasturtium officinale), perfazendo um total de 200 amostras.

A técnica de análise das amostras, empregada no presente estudo, foi realizada de acordo com Oliveira e Germano ${ }^{7}$ (1992).

Foi utilizada a distribuição $\mathrm{T}$ de Student para o cálculo do teste de duas propotçōes, com aproximação normal, a fim de comprovar ou não a existência de diferenças significantes entre os níveis de contaminação das quatro variedades de hortaliças analisadas, em relação aos cistos de protozoários encontrados.

Tabela 1. Hortaliças amostradas, comercializadas na CEAGESP, segundo as variedades de amostras $\theta 0$ re. sultado laboratorial da pesquisa de protozoários. São Paulo, 1991

\begin{tabular}{cccccccc}
\hline $\begin{array}{c}\text { Variedade } \\
\text { de }\end{array}$ & \multicolumn{2}{c}{$\mathrm{P}$} & \multicolumn{2}{c}{$\mathrm{N}$} & \multicolumn{2}{c}{ Total } \\
\cline { 2 - 8 } amostra & $\mathrm{F}$ & $\%$ & $\mathrm{~F}$ & $\%$ & $\mathrm{~F}$ & $\%$ \\
\hline Alface lisa & 9 & 18,0 & 41 & 82,0 & 50 & 100,0 \\
Alface crespa & 13 & 26,0 & 37 & 74,0 & 50 & 100,0 \\
Escarola & 17 & 34,0 & 33 & 66,0 & 50 & 100,0 \\
Agrião & 30 & 60,0 & 20 & 40,0 & 50 & 100,0 \\
\hline
\end{tabular}

CEAGESP - Companhia de Entrepostos e Armazéns Gerais de São Paulo
$P=$ Positivo
$N=$ Negativo

Adotou-se, como nível de rejeição, alfa igual a 0,05 e o valor de $\mathrm{Z}$ de alfa igual a 1,96 (Berquó e col. ${ }^{2}, 1981$ ).

\section{Resultados}

Os níveis de contaminação por protozoários, obtidos para as quatro variedades de hortaliças variaram de $18,0 \%$ para a alface lisa, a $60,0 \%$ nas amostras de agrião (Tabela 1).

Através do teste de duas proporções, observou-se diferenças estatisticamente significantes entre as amostras de agriāo e alface lisa $(Z=4,31)$, entre agrião e alface crespa $(Z=$ $3,43)$ e agrião e escarola $(Z=2,60)$.

A análise das freqüências de cada tipo de protozoário (Tabela 2) evidenciou uma predominância na ocorrência de cistos de Entamoeba $s p$ (com 4 e 8 núcleos) e de Giardia $s p$, em todas as variedades de hortaliças. No entanto, os cistos de Endolimax $s p$ apresentaram freqüência elevada apenas nas amostras de agrião. Outros parasitas recuperados, com menor freqüência, incluíram lodamoeba sp e Chilomastix $s p$.

As amostras de agriāo revelaram as maiores freqüências de ocorrência para todos os tipos de protozoários, enquanto que as de alface lisa foram as que apresentaram os niveis mais baixos de contaminação.

O teste de duas proporções, aplicado aos valores apresentados na Tabela 2 , revelou diferenças estatisticamente significantes entre os percentuais de contaminação da alface lisa e crespa, apenas com relação à freqüência de

Tabela 2. Hortaliças amostradas, comercializadas na CEAGESP, segundo as variedades de amostras e os resul. tados laboratoriais da pesquisa de protozoários. São Paulo, 1991.

\begin{tabular}{|c|c|c|c|c|c|c|c|c|}
\hline \multirow{2}{*}{$\begin{array}{c}\text { Tipo } \\
\text { de } \\
\text { protozoário }\end{array}$} & \multicolumn{2}{|c|}{$\begin{array}{c}A L \\
n=50 \\
(100 \%)\end{array}$} & \multicolumn{2}{|c|}{$\begin{array}{c}A C \\
n=50 \\
(100 \%)\end{array}$} & \multicolumn{2}{|c|}{$\begin{array}{c}\text { ES } \\
n=50 \\
(100 \%)\end{array}$} & \multicolumn{2}{|c|}{$\begin{array}{c}A G \\
n=50 \\
(100 \%)\end{array}$} \\
\hline & $F$ & $\%$ & $\mathbf{F}$ & $\%$ & $F$ & $\%$ & $\mathbf{F}$ & $\%$ \\
\hline Entamoeba sp (4N) & 4 & 8,0 & 7 & 14,0 & 5 & 10,0 & 13 & 26,0 \\
\hline Entamoeba sp (8N) & 0 & 0,0 & 5 & 10,0 & 8 & 16,0 & 11 & 22,0 \\
\hline Giardia sp & 2 & 4,0 & 5 & 10,0 & 6 & 12,0 & 12 & 24,0 \\
\hline Endolimax sp & 2 & 4,0 & 3 & 60,0 & 0 & 0,0 & 17 & 34,0 \\
\hline lodamoeba sp & 0 & 0,0 & 1 & 2.0 & 4 & 8,0 & 4 & 8,0 \\
\hline Chilomastix sp & 0 & 0,0 & 0 & 0,0 & 0 & 0,0 & 2 & 4,0 \\
\hline
\end{tabular}

CEAGESP - Companhia de Entrepostos e Armazéns Gerais de São Paulo

$A L=$ alface lisa

$A C=$ alface crespa

ES = escarola

$A G=$ agriāo $\mathrm{n}$ = número de amostras

$F=$ freqüência

$\%=$ percentagem 
Entamoeba $s p$, com 4 núcleos $(\mathrm{Z}=2,30)$; de alface lisa e escarola, com relação às freqüências de Entamoeba $s p$, com 8 núcleos $(\mathrm{Z}=$ $2,95)$ e Iodamoeba sp $(Z=2,04)$; de alface lisa e agriāo, com relação a todos os tipos de protozoários, a exceção de Chilomastix sp; de alface crespa e agriẫo, em relação à contaminação por cistos de Endolimax sp $(Z=3,50)$; $e$, de escarola e agrião, em relação à contaminação por Entamoeba $s p$, de 4 núcleos $(\mathrm{Z}=$ 2,08) e Endolimax $s p(Z=4,53)$.

Não houve diferenças estatisticamente significantes entre os níveis de contaminação apresentados nas amostras de alface crespa e escarola.

\section{Discussão e Conclusões}

Os resultados obtidos revelaram elevados percentuais de contaminação por protozoários, em todas as variedades de hortaliças estudadas. As freqüências de cistos foram, significativamente, maiores no agrião, seguido da escarola, alface crespa e alface lisa (Tabela 1). Esta seqüência já havia sido observada em estudo anterior ${ }^{7}$, com relação aos níveis de contaminação por helmintos, o que reforça a importância da estrutura vegetal no grau de contaminação de hortaliças. Assim, o agrião, com folhas múltiplas e separadas, permite maior fixação dos cistos, ao contrário da alface, cujas folhas largas e firmemente justapostas dificultam a aderência. A escarola, possuindo características físicas intermediárias, apresenta níveis de contaminação situados entre aquelas duas variedades.

Há uma relação inversa entre os percentuais de contaminação e a qualidade das condições sanitárias do ambiente e as práticas de plantio utilizadas ${ }^{6}$. Assim, alguns autores ${ }^{1,3}$ admitem que as culturas de alface recebem cuidados mais adequados por parte dos horticultores, o que pode ter contribuído para a obtenção de percentuais de contaminação relativamente menores nessas hortaliças. Os cistos de protozoários sobrevivem por períodos de tempo mais prolongados no meio aquático. Este fato poderia justificar as freqüências superiores, sobretudo de Endolimax sp, encontradas no agrião, cujo cultivo exige terrenos permanentemente inundados.

Os protozoários recuperados apresentam importância para a saúḋ pública, pois indicam contaminação fecal de origem humana e/ou animal, tal como ocorre com Entamoeba $s p$ (4 núcleos), Giardia sp e Iodamoeba $s p$ que apresentam espécies de ocorrência no homem, nos animais ou em ambos ${ }^{1,9}$.

Dentre os protozoários mais freqüentemente encontrados destacou-se a Giardi $s p$, cuja prevalência na população residente na Região Metropolitana de São Paulo é igualmente elevada, o que evidencia a contaminação de origem humana ${ }^{11}$.

Os cistos de Entamoeba sp, de 4 núcleos, característicos de Entamoeba histolytica, não se distinguem morfologicamente dos cistos de Entamoeba moshkovskii ${ }^{9}$, amebídeo de vida livre que normalmente habita os esgotos, o que poderia justificar as altas freqüências obtidas (Tabela 2). Contudo, algumas amebas de vida livre, tais como Acanthamoeba e Naegleria, cuja transmissão é favorecida pela exposição às águas contaminadas por esgotos, são apontadas como causadoras de meningoencefalites no homem ${ }^{12}$. Por esta razão, embora estes gêneros não tenham sido observados, é possivel que as hortaliças analisadas, notadamente 0 agrião, apresentem importância considerável na epidemiologia dessas enfermidades ${ }^{10}$.

Outros protozoários recuperados, tais como Endolimax sp e Chilomastix sp, são exclusivos do homem ${ }^{9}$ e comprovam a ocorrência de contaminação por fezes humanas. Os cistos de Entamoeba sp., de 8 núcleos, característicos de Entamoeba coli, bem como Endolimax nana e Chilomastix mesnili, embora não se disponha de dados específicos, apresentam, possivelmente, niveis de ocorrência elevados na população humana da Regiāo $\mathrm{Me}$ tropolitana de São Paulo ${ }^{9}$. Embora não sejam considerados patogênicos, estes protozoários apresentam grande valor como indicadores de contaminação fecal de origem humana nas hortaliças. Por esta razão, recomenda-se que os exames parasitológicos sejam realizados totineiramente, ao lado da colimetria, como instrumento auxiliar para o monitoramento sanitário desses produtos.

Considerando-se a magnitude dos resultados obtidos no presente estudo, bem como o risco à saúde que as hortaliças podem apresentar, quando contaminadas por protozoários intestinais, ressalta-se a necessidade da adoção de medidas que propiciem uma melhoria do quadro apresentado. Deste modo, as atividades de vigilância sanitária devem ser concentradas na produção das hortaliças, através de ações educativas destinadas aos produtores, e do monitoramento laboratorial das águas destinadas à irrigação das hortas. 
A desinfecção das hortaliças, previamente ao consumo, pode apresentar relevância considerável no sentido de minimizar os riscos de transmissão de enteroparasitoses através desses alimentos, uma vez que a lavagem simples não reduz a contaminação por cistos.

Um método simples e eficaz, tanto para os cistos de protozoários, quanto para ovos de helmintos, consiste na imersão das folhas em água aquecida a $60^{\circ} \mathrm{C}$, por dez minutos. Este procedimento, pela maior facilidade de execução em nível doméstico, deve ser especialmente considerado na formulação de programas educativos direcionados à população consumidora desses alimentos.

OLIVEIRA, C.A.F. de \& GERMANO, P.M.L. [Study of the occurrence of intestinal parasites on vegetables commercially traded in the metropolitan area of S.Paulo, SP - Brazil. II - Research into protozoan cysts]. Rev.Saúde públ., S.Paulo, 26: $332-5,1992$. Vegetables in natura, commercially traded in the metropolitan area of S.Paulo, Brazil, were analised by means of the appropriate methodology with a view to discovering and identifying protozoan cysts of medical interest. The vegetables under study consisted of 50 samples of each of the variaties listed below: lettuce (Lactuca sativa) - oily leaves and crisphead varieties, endive (Chicorium sp) and water-cress (Nasturtium officinale). Results showed high rates of contamination in all the varieties of vegetable analysed. However, the water-cress was the one which presented the highest frequencies of enteroparasites. Both the oily leafes and crisphead varieties of lettuce presented the lowest rates of contamination, whereas endive presented values ranking, in general, between those of the lettuce and those of the water-cresses. A great variety of those protozoans which occur frequently in the population resident in the metropolitan area of $\mathbf{S}$. Paulo were observed in the samples, the most frequent being Entamoeba $s p$ (with 4 and 8 nuclei) and Giardia sp. Cysts of Iodamoeba sp, Endolimax $s p$ and Chilomastix $s p$ were also recovered from the samples, thus corroborating the occurrence of high rates of fecal contamination. The significance of these kinds of food in the transmission of protozoans is discussed in the light of the results obtained.

Keywords: Vegetable, analysis. Protozoan, isolation. Food contamination, analysis.

\section{Referências Bibliográficas}

1. ACHA, P.N. \& SZYFRES. B. Zoonosis y enfermedades transmissibles comunes al hombre y a los animales. 2' ed. Washington, D.C., Organización Panamericana de la Salud, 1986 (OPAS Publicación Cientifica, 503)

2. BERQUO, E.S.; SOUZA, J.M.P.; GOTLIEB, S.L.D. Bioestatistica. São Paulo, EPU, 1981.

3. FEACHEM, R.G.; BRADLEY, D.J.; GARELICK, H.; MARA,D. Sanitation and disease: health aspects of excreta and wastewater management. Chichester, John Wiley and Sons, 1983.

4. HERNANDES, N.; CIMERMAN, B.; FERNANDES, M.F.P.; FERRAZ, C.A.M.; ARAUJO, A.L; SILVA, C.A. Estudo da contaminação de ventura no Municipio de Biritiba Mirim. In: Congresso Brasileiro de Parasitologia, 69, Belo Horizonte, 1981. Resumos. Belo Horizonte, Sociedade Brasileira de Parasitologia, 1981. p. 219.

5. MICHE, M.P. \& MORGANTI, LE Parasitas em alfaces comercializadas na cidade de São Paulo. In: Congresso da Federación Latinoamericana de Parasitólogos, 6\% Congresso da Sociedade Brasileira de Parasitologia, 82/ Jomada Paulista de Parasitologia, 52, São Paulo, 1983. Resumas. São Paulo, Sociedade Brasileira de Parasitologian 1983. p. 120.

6. MOTA C.C.S.; ELIAS, A.; MIKOSZEWSKA, I.; VIEIRA, H.RA.; PICHET NETO, J.; VASQUES, RM.R; ALMEIDA,A.A.; GAISSLER, M.S.; BEATRIZ, R; MOTA, R.M.T.C.S. Condiçōes higiênico-sanitárias de hortaliças comercializadas em Curitiba-PR (Brasil). In: Congresso Brasileiro de Ciência e Tecnologia de Alimentos, 60 , Brasília, 1983. Programa e resumos. Brasília, Sociedade Brasileira de Ciência e Tecnologia de Alimentos, 1983. p. 125.

7. OLIVEIRA, C.A.F. \& GERMANO, P.M.L. Estudo da ocorrência de enteroparasitas em hortaliças comercializadas na Região Metropolitana de São Paulo - SP, Brasil. I - Pesquisa de helmitos. Rev. Saúde públ. S.Paulo, 26: 283-9, 1992.

8. ORGANIZACION MUNDIAL DE LA SALUD. Comite de Expertos en Prevención y Control de Infecciones Parasitarias Intestinales, Ginebra, 1986. Informe. Ginebra, 1987. (Serie de Informes Técnicos, 749)

9. PESSOA, S.B. \& MARTINS, A.V. Pessóa parasitologia médica. $11^{2}$ ed. Rio de Janeiro, Guanabara Koogan, 1982.

10. RUDE, R.A.; JACKSON, G.J.; BIER, J.W.; SAWIER, T.K.; RISTY, N.G. Survey of fresh vegetables for nematodes, amoebae and Salmonella. J.Ass.off.anal.Chem., 67:613-5, 1984.

11. WALDMAN, E.A. \& CHIEFFI, P.P. Enteroparasitoses no Estado de São Paulo: questão de saúde pública. Rev. Inst. Adolfo Lut S.Paulo, 49: 93-9, 1989.

12. WARHURST, D.C. Pathogenic free-living Amoebae. Parasit. Today, 1: 24-8, 1985.

Recebido para publicação em 9.1.1992 Reapresentado em 19.3.1992

Aprovado para publicação em 24.5.1992 